\title{
"Transverse" persistent currents in mesoscopic cylinders and rings
}

\author{
Igor O. Kulik
}

Department of Physics, Bilkent University, Ankara 06533, Turkey

\begin{abstract}
Persistent current is a nondecaying current in a normal metal ring or cylinder induced by the vector potential applied parallel to the direction of the current (longitudinal, or Aharonov-Bohm persistent current). A magnetic field applied perpendicular to the wall of the hollow metallic cylinder also produces an azimuthal nondecaying current (a transverse persistent current) even in the absence of the Aharonov-Bohm flux. Magnetic moment of a single connected conductor (a $2 \mathrm{~d}$ conducting stripe) is supported by a current (a Landau-persistent current) which is shown to oscillate in space. (C) 2000 Published by Elsevier Science B.V. All rights reserved.
\end{abstract}

Keywords: Aharonov-Bohm effect; Flux quantization; Persistent current

Persistent currents in mesoscopic systems (cylinders, rings) first predicted by the author [1] and later rediscovered by Buttiker et al. [2] arise due to the Aharonov-Bohm phase [3] accumulated by electron in its motion around the circumference of the ring. The current is periodic function of the magnetic flux in the ring with a period of flux quantum $h c / e$. The current does not decay in time since it corresponds to the minimum of system energy in the presence of the azimuthal component of the vector potential $A_{\varphi}$, i.e. the ground state of system is a current-carrying one. We investigate in this paper another sources of persistent currents, those related to Landau diamagnetism in a single connected body (Landau persistent current), and to transverse component of the magnetic field in the hollow metallic cylinder. Landau current is calculated [4] in a $2 \mathrm{~d}$ metallic stripe (of width $d$ ) perpendicular to magnetic field $B$. We assume that $d$ is much smaller than the electron cyclotron radius $r_{\mathrm{c}}$ which makes the current nonvanishing in all sample and oscillating as a function of position. The oscillations preserve at temperature below the degeneracy temperature $T_{\mathrm{F}}$ while even at $T \geqslant T_{\mathrm{F}}$, the current remains nonzero and nondecaying in time. Such an effect

E-mail address: kulik@fen.bilkent.edu.tr (I.O. Kulik) was understood quite long ago by Teller [5], who however considered the opposite limit of $d \gg r_{\mathrm{c}}$.

In a double-connected geometry shown in Fig. 1, a state of the system (double coupled rings (a) or hollow cylinder (b) in the presence of both longitudinal and transverse flux) is specified by two independent variables, $A_{\varphi}^{0}=\Phi_{1} / L$, and the radial field, $B_{\mathrm{r}}=\Phi_{\mathrm{t}} / L h(L$ is the circumference and $h$ the height), in such a way that the energy of the ground state is a nonmonotonous function of the longitudinal and transverse fluxes. In case of a double ring, assuming the tight-binding model for electron transport, system Hamiltonian reads

$$
\begin{aligned}
H= & -\sum_{n=1}^{N}\left(t_{1} a_{n}^{+} a_{n+1} \mathrm{e}^{\mathrm{i}(\alpha+\beta)}+t_{2} b_{n}^{+} b_{n+1} \mathrm{e}^{\mathrm{i}(\alpha-\beta)}\right) \\
& +h c-t_{12} \sum_{n=1}^{N}\left(a_{n}^{+} b_{n}+b_{n}^{+} a_{n}\right),
\end{aligned}
$$

where $a_{n}^{+}\left(a_{n}\right)$ creates (annihilates) electron in the upper and $b_{n}^{+}\left(b_{n}\right)$ in the lower ring, and $t_{1}, t_{2}, t_{12}$ are the corresponding hopping amplitudes. Phase $\alpha$ is related to longitudinal, and phase $\beta$ to transverse flux according to $\alpha=2 \pi \Phi_{1} / N \Phi_{0}, \beta=\pi \Phi_{\mathrm{t}} / N \Phi_{0}$ where $N$ is the number of sites. Derivative of system energy with respect to $\alpha$ gives AB-persistent current, and the derivative with respect to $\beta$, the Landau current. Electron energy in 


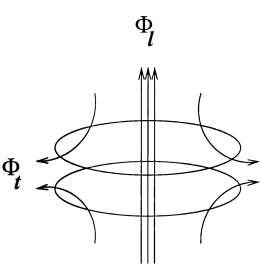

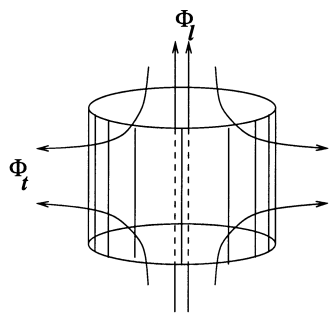

b
Fig. 1. Double ring (a) and hollow cylinder (b) subject to the longitudinal $\left(\Phi_{1}\right)$ and to transverse $\left(\Phi_{\mathrm{t}}\right)$ fluxes.

a double ring is

$\varepsilon_{k}=-t_{1} \cos (k+\alpha+\beta)-t_{2} \cos (k+\alpha-\beta)$

$$
\pm \sqrt{\left(t_{1} \cos (k+\alpha+\beta)-t_{2} \cos (k+\alpha-\beta)\right)^{2}+t_{12}^{2}}
$$

where $k=2 \pi n / N, n=0, \pm 1, \ldots$.

The circular current $J$ is calculated as a derivative of the system free energy with respect to longitudinal flux $\Phi_{1}$. The nonzero current at $\Phi_{1}=0$ appears once system is not central symmetric, e.g. in the case of nonequal hopping amplitudes in the rings $\left(t_{1} \neq t_{2}\right)$ (This is equivalent to nonequal effective masses of carriers in two rings.)

Same treatment can be applied to a cylinder assuming a two-dimensional network of sites with operators $a_{n m}^{+}$creating electrons at the sites. We also add random potential at sites $V_{n m}=V_{\text {rnd }} \cdot \xi_{n m}$ where $\xi_{n m}$ is a random variable satisfying $-1<\xi_{n m}<1$. The central symmetry is now violated by the randomness of scattering events of electrons at sites. Fig. 2 shows a result of computer simulation for "transverse" component of the current in absence of the longitudinal flux, as a function of radial flux $\Phi_{\mathrm{t}}$. The chaotic quasi-oscillatory behavior is hard to interpret quantitatively, it corresponds to flux quantization in the local "loops" which may be formed due to impurity inhomogeneity. Actually, the dependence, if properly inverted to scattering center distribution, can be

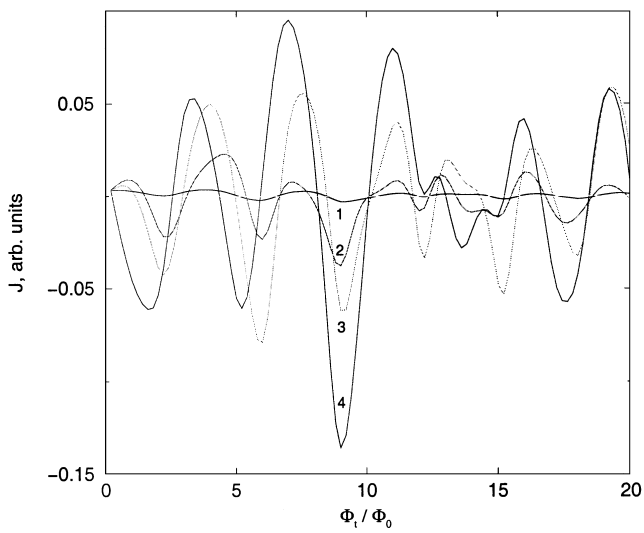

Fig. 2. Azimuthal current in the disordered cylindrical shell with $10 \times 10$ sites vs transverse flux $\Phi_{\mathrm{t}}$ at $\Phi_{1}=0$ and at various disorder amplitudes. The curves are labeled such that (1) $V_{\text {rnd }} / t=0$, (2) $V_{\text {rnd }} / t=0.05$, (3) $V_{\text {rnd }} / t=0.1$, (4) $V_{\text {rnd }} / t=0.15$. The small nonzero value of $J$ at $V_{\text {rnd }}=0$ arises due to numeric evaluation of the derivative $\partial F / \partial \Phi_{1}$ at small but nonzero $\Phi_{1}$.

served as an information on the inhomogeneous state of mesoscopic system. We also mention that the transverse current may contribute to an experimental observation of longitudinal persistent current [6] and in particular we may consider whether it can substantially increase the amplitude of the Aharonov-Bohm oscillation.

\section{References}

[1] I.O. Kulik, JETP Lett. 11 (1970) 275.

[2] M. Buttiker, Y. Imry, R. Landauer, Phys. Lett. A 96 (1983) 365.

[3] Y. Aharonov, D. Bohm, Phys. Rev. 115 (1959) 485.

[4] I.O. Kulik, Bull. Am. Phys. Soc. 44 (1) (1999) 222.

[5] E. Teller, Zs. Phys. 67 (1931) 311.

[6] V. Chandrasekhar, R.A. Webb, M.J. Brady, M.B. Ketchen, W.J. Gallagher, A. Kleinzasser, Phys. Rev. Lett. 67 (1991) 3578 . 